American Journal of Pharmaceutical Education 2016; 80 (8) Article 131.

\title{
RESEARCH
}

\section{Effect of Communication Style on Perceptions of Medication Side Effect Risk among Pharmacy Students}

\author{
Ruta V. Sawant, MS, Collin R. Beatty, PharmD, MS, Sujit S. Sansgiry, PhD \\ University of Houston College of Pharmacy, Houston, Texas \\ Submitted July 31, 2015; accepted November 20, 2015; published October 25, 2016.
}

Objective. To assess the effect of communication style, and frequency and severity of medication sideeffects, on pharmacy students' perception of risk of experiencing side effects.

Methods. One hundred responses from pharmacy students were obtained using an online survey. Participants were presented with a drug information box containing drug name, drug usage, and one side-effect associated with the drug. Information on side-effect for each drug was presented in one of eight experimental conditions, in a 2 (side-effect frequency: low, high), X2 (side-effect severity: mild, severe) X2 (communication style: verbal, verbal + natural frequency) factorial design. Risk perception of experiencing side effects was measured.

Results. Communication style was found to have a significant impact on risk perception depending on the context of frequency and severity associated with the side effect.

Conclusion. Communication style plays a significant role in formulating risk perceptions of medication side effects. Training in pharmaceutical counseling should include special emphasis on effective language use.

Keywords: Risk communication, risk perception, medication side-effects risk, verbal and numeric risk, pharmacy students

\section{INTRODUCTION}

Fear of potential side effects is reported to be the single greatest deterrent from taking prescription drugs. ${ }^{1}$ Although information on medication side effects is the most frequently requested information by patients, it has detrimental effects on the evaluations of medications and medication adherence. ${ }^{2}$ Thus, providing information about side effects in a manner that leads to correct interpretation of the intended message is of great value. Research indicates that use of semantic or verbal descriptors (ie, use of expressions like "one may rarely experience" or "most common side effect") to express probability of a side effect has more effect on the estimates of risk and on treatment advice when compared to numeric expressions. ${ }^{3,4}$ However, there is wide variability in interpretation of verbal expressions and, when patient interpretation differs from that of health care providers, compliance problems may arise. ${ }^{5}$ Verbal descriptions may be considered vague and difficult to interpret. Numeric descriptors like percentages (50\% chance) or natural frequencies (4 in 100)

Corresponding Author: Sujit S. Sansgiry, Department of Pharmaceutical Health Outcomes and Policy,University of Houston College of Pharmacy, 1441 Moursund St., Houston, TX 77030. Tel: 832-842-8392. Fax: 713-795-8383.

E-mail: ssansgiry@uh.edu are considered easier to understand by physicians and patients. ${ }^{6,7}$ In fact, patients prefer numeric information to verbal descriptions about frequency of side effects. ${ }^{8}$

Information about medication side effects is often provided to patients during counseling sessions with pharmacists. For effective and accurate patient education to be provided, pharmacists need knowledge and skills of patient counseling. Messages communicated to patients need to be adapted to suit patients' language skills and understanding level. ${ }^{9}$ Foundations of counseling can be laid during the formal years of pharmacy education. Pharmacy students are trained in counseling through practical examinations and real-world experiences behind the counter. During these formative years, students should be made aware of intricacies involved in communicating risks of medication side effects. Self-perceptions and characteristics of a particular behavior can also have an influence on the manner in which a message is communicated to patients. A study by Antworth and colleagues demonstrated that over or underweight health care providers were less confident in counseling patients regarding weight and lifestyle modification compared to their normal weight counterparts. ${ }^{10}$ Ahmed et al also reported that lack of knowledge and understanding of a disease and related concerns is associated with lower confidence in 


\section{American Journal of Pharmaceutical Education 2016; 80 (8) Article 131.}

counseling competence. ${ }^{11}$ Such instances may also arise with respect to confidence in counseling on the risk of medication side effects. As there are no formal structures regarding use of language and terms in counseling medication side effect risk, pharmacy students may use semantics based on their own perceptions. Even though they may intend to communicate one particular value of frequency of side effect, it may not be translated correctly by the receiver (patient), leading to misinterpretation. Thus, the purpose of a counseling session may not be adequately served. The use of vague verbal descriptors is reported among pharmacists, ${ }^{12}$ further supporting the need to train pharmacy students appropriately in communicating medication side effects.

To identify focus areas of training for communicating risk of medication side effects, we need to understand perceptions of pharmacy students when presented with commonly used verbal and numeric descriptors. No study has yet investigated how pharmacy students perceive these verbal descriptors and whether perceptions differ when verbal descriptors are combined with numbers. Assessing pharmacy students' perception of risk of side effects can give insight into their understanding of commonly used language in counseling sessions, which may eventually help in future educational interventions to improve pharmacist-patient communications. Another important factor that may alter perceptions of risk is the context in which the risk is communicated. Interpretation of risk information is influenced by the contextual effects of perceived severity and perceived base rate of the medication side effect. ${ }^{13}$ Thus, the current study involved assessing perceptions of risk of experiencing side effects in scenarios with three interacting factors: (1) communication style (verbal descriptors vs verbal and numeric descriptors); (2) frequency (probability of occurrence); and (3) severity of side effect.

\section{METHODS}

Participants were recruited through an online survey administered to University of Houston College of Pharmacy students using Qualtrics software (Qualtrics, Provo, UT). Invitations were e-mailed via college listserv groups to all pharmacy students. Approval from University of Houston Committee for the Protection of Human Subjects was obtained. Participants were presented with information on four drugs in the form of a drug information box for each drug. The drug information box consisted of drug name (labeled as Drug A, B, C, or $\mathrm{D}$ to avoid any potential bias resulting from previous knowledge about currently available medications), drug usage, and one side effect associated with the drug. Information on a side effect for each drug was presented in one of eight experimental combinations, in a 2 (side effect frequency: low, high) X2 (side effect severity: mild, severe) X2 (communication style: verbal, verbal combined with natural frequency) factorial design. Information from package inserts and an extensive literature review was used to develop statements about side effects. Information in the verbal format was provided as "Drug $[\mathrm{X}]$ can $[\mathrm{Y}]$ cause $[\mathrm{Z}]$ ". Information in the verbal combined with natural frequency format was provided as "Drug $[\mathrm{X}]$ can $[\mathrm{Y}]$ cause $[\mathrm{Z}]$. Out of 100 people taking Drug $[\mathrm{X}],[\mathrm{W}]$ will experience [Z]." $\mathrm{X}$ represented the drug name (eg, A-D); Y represented the verbal risk statement (eg, rarely, very likely); $\mathrm{Z}$ represented the side effect associated with the drug (eg, headache); and $\mathrm{W}$ represented the natural frequency of side effect (eg, 4 out of 100).

The severity and frequency of side effects for the four drugs were selected as specified in Table 1. To validate the selected severity of the side effects, participants were requested to rank each side effect from most to least severe. Side effects ranked "1" were perceived as "severe" side effects, and those ranked "4" were perceived as "mild" side effects. Each participant received information about four medication side effects (one for each drug), where the descriptions were randomized to either verbal or the combined communication style with either a high or low frequency and a mild or severe associated severity with the side effect. An example of a drug information box is provided in Appendix 1.

Frequency (low vs high), severity (mild vs severe), and communication style (verbal vs verbal combined with numeric descriptors) for side effects were the primary independent variables in the model. Perceptions of risk of experiencing medication side effect (ie, risk perception) based on information presented in the drug information was the primary dependent variable and was measured using the question: "If you took the medication, what do you think is your risk of experiencing the side effect?" A Visual Analog Scale (VAS) ranging from 0 to 100 followed the question for recording responses regarding risk perception. Higher scores on the VAS indicated higher perceived risk.

Before presenting participants with the drug information boxes, a general risk assessment score (labeled as general risk perception) was obtained. This score measured an individual's general perception about risk associated with taking any medications for any conditions and was captured by asking the question, "How risky do you believe it is in general to take medications for any condition?" (VAS ranging from 0 to 100). Finally, demographic characteristics such as age, gender, ethnicity, and level of education were measured. 


\section{American Journal of Pharmaceutical Education 2016; 80 (8) Article 131.}

Table 1. Drugs Side Effects, Frequencies, and Severities Used for Drug Information Box

\begin{tabular}{llcll}
\hline Drug & Side effect (Severity) & Natural Frequency (Out of 100) & Severity & Verbal Descriptions \\
\hline A & Stomach bleeding & 2 & Severe & Rarely \\
B & Facial flushing & 5 & Mild & Rarely \\
C & Loss of immune function & 85 & Severe & Very likely \\
D & Headache & 88 & Mild & Very likely \\
\hline
\end{tabular}

All analyses were conducted using SAS, v9.3 (SAS Institute Inc., Cary, NC). Analysis of covariance (ANCOVA) was used to assess the effects of communication style, frequency, and severity on risk perception while adjusting for general risk perception, age, gender, education, and race. For ANCOVA, general risk perception and age were taken as continuous variables whereas gender, education (classified as " $\leq 2$-year college degree" as one category and " $\geq 4$-year college degree" as a second category) and race (classified as white and nonwhite) were included as categorical variables. Sample size calculated using G*Power v3.1 (Heinrich Heine Universität Düsseldorf, Germany) at a significance level of 0.05 , power of 0.80 , and effect size of 0.25 indicated that a sample of 100 was adequate for the analyses. Descriptive statistics were obtained by calculating means for continuous variables and frequencies for categorical variables. Mean risk perception scores were obtained for eight possible combinations of the three factors of communication style, frequency, and severity. Post hoc Tukey-Kramer test was conducted for pairwise comparisons of mean risk perception scores of all eight combinations. All statistical analysis were performed at an a priori significance level of 0.05 .

\section{RESULTS}

One hundred completed surveys were obtained. Participants' age ranged from 20 to 39 with a mean of 25 $(\mathrm{SD}=3)$. Seventy-three percent of participants were female students. Most participants were either white (53\%) or Asian $(38 \%)$, with a small number of Hispanics (4\%) and African Americans (1\%). With respect to education level, about $26 \%$ had some college degree, $62 \%$ had a bachelor's degree, and $12 \%$ had a master's or higher level degree. The general risk perception ranged from 0 to 85 with an average of 41 (18). When asked to rank the side effects in the order of their severity from 1 to $4(1=$ mild, $4=$ severe), participants correctly identified stomach bleeding (mean rank =3.2) and loss of immune function (mean rank =3.7) as severe side effects. Headache (mean rank=1.6) and flushing of the face (mean rank=1.4) were correctly ranked as mild side effects. This indicated that the simulations used for severity of side effects were valid.

Means were obtained for the eight combinations of communication style, frequency, and severity of side effects (Table 2). Overall low frequency side effects (1 to 4) had lower risk perception scores (range: 12 to 13) compared to high frequency side effects (5 to 8) (range: 57 to 82). Further, ANCOVA was used to test independent and interaction effects of these means on risk perception.

A $2 \times 2 \times 2$ factorial ANCOVA (Table 3) was performed to test the effects of communication style, frequency, and severity on risk perception while controlling for general risk perception, age, race, gender, and education level (each of the 100 participants provided responses for four drug information boxes yielding a total $\mathrm{N}$ of 400$)$. Frequency $(\mathrm{F}=1260, p<0.0001)$, severity $(\mathrm{F}=19, p<0.0001)$ and communication style $(\mathrm{F}=5.67$, $p=0.02$ had significant main effects on risk perception (Table 3). Among all the covariates, only general risk perception about medication side effects was found to be significantly associated with the risk perception of side effects specific to the information provided $(\mathrm{F}=15$, $p=0.0002$ ). 2- and 3-way interaction effects between communication style, frequency, and severity were also significant.

To better understand the interaction effect and combined effects, we conducted post hoc Tukey-Kramer test for pairwise comparisons between the means of different combinations of the three factors (Table 2). To make interpretation easier, we looked at simple 2-way interaction of frequency and severity by communication style as provided in Figure 1. Further post hoc comparison results can be seen in Figure 2.

Figure 1 (A and B) shoes that low frequency side effects were associated with lower risk perception scores and high frequency side effects were associated with higher scores. At the same time, difference in risk perception scores between verbal and combined communication style were dependent on the severity of side effects. In the case of mild side effects (Figure 1A), verbal and combined communication styles had significantly different risk perception scores when the frequency of side effects was high [mean difference between verbal and combined $=25$; CI $(14,36) ; p<0.0001]$. On the other hand, among severe side effects (Figure 1B), verbal and combined communication styles had different risk perception scores when the frequency of side effects was low [mean difference between verbal and combined $=7$; 
American Journal of Pharmaceutical Education 2016; 80 (8) Article 131.

Table 2. Mean Risk Perception Scores for Communication Style, Frequency, and Severity of Side Effects

\begin{tabular}{llllrr}
\hline$\#$ & Communication Style & Frequency & Severity & Risk Perception Mean (SD) & Range \\
\hline 1 & Verbal & Low & Mild & $13(14)$ & $0-67$ \\
2 & Combined & Low & Mild & $12(15)$ & $2-69$ \\
3 & Verbal & Low & Severe & $19(16)$ & $0-73$ \\
4 & Combined & Low & Severe & $12(15)$ & $1-60$ \\
5 & Verbal & High & Mild & $57(29)$ & $0-97$ \\
6 & Combined & High & Mild & $82(16)$ & $6-100$ \\
7 & Verbal & High & Severe & $81(16)$ & $30-100$ \\
8 & Combined & High & Severe & $81(15)$ & $10-100$ \\
\hline
\end{tabular}

CI $(-3,18) ; p=0.40]$. Therefore, one can conclude that the interaction effect was mainly a result of the complexity of understanding the severity factor.

Figure 1 (A and B) also suggest that the slopes of lines for combined communication style are steeper compared to those for verbal communication style. This indicates that differentiation among low and high frequency side effects was better when combined communication style was used instead of verbal communication style. When verbal communication style was used, the difference in risk perception scores between low and high frequency side effects was 43 [CI $(33,54)$; $p<0.0001]$ for mild side effects and 62 [CI $(52,73)$; $p<0.0001]$ for severe side effects. On the other hand, when combined communication style was used, the difference in risk perception scores between low and high frequency side effects was 70 [CI $(59,80) ; p<0.0001]$ for mild side effects and 69 [CI $(59,80) ; p<0.0001$ ] for severe side effects.

\section{DISCUSSION}

Findings from the study indicated that communication style, frequency, and severity had significant impact on risk perception of side effects among pharmacy students. Overall, high frequency side effects had higher risk perception scores compared to low frequency side effects, and severe side effects had higher risk perception scores compared to mild side effects, as anticipated. However, the impact of communication style on risk perception varied depending on the levels of frequency and severity. When low frequency + mild severity or high frequency + severe severity were evaluated, risk perceptions were not greatly affected by change in the communication style used. This may be because arriving at an interpretation for these two combinations was not as difficult because of the extreme nature of both contexts. However, in the case of low frequency + severe severity or high frequency + mild severity, interpretation of risk may require additional cues (ie, communication style) because of the complex nature of the context. Although a low frequency side effect may be perceived as less risky, the severe nature of the same side effect may make interpretation of risk difficult. In such instances, presenting the exact numeric information (as in combined communication style) may help to understand the underlying risk, as demonstrated in our study. Providing information in a combined communication style helped

Table 3. Analysis of Covariance for the Effect of Frequency, Severity, and Communication Style on Risk Perception of Experiencing Side Effects ${ }^{\mathrm{a}}$

\begin{tabular}{|c|c|c|c|c|c|}
\hline Variable & DF & Sum of Squares & Mean Square & $F$ value & $p$ value \\
\hline Frequency $(\mathrm{F})$ & 1 & 371918 & 371918 & 1260 & $<.0001$ \\
\hline Severity (S) & 1 & 5494 & 5494 & 19 & $<.0001$ \\
\hline Communication style (C) & 1 & 1673 & 1673 & 6 & 0.018 \\
\hline $\mathrm{F} \times \mathrm{S}$ & 1 & 2161 & 2161 & 7 & 0.007 \\
\hline $\mathrm{F} \times \mathrm{C}$ & 1 & 8281 & 8281 & 28 & $<.0001$ \\
\hline $\mathrm{S} \times \mathrm{C}$ & 1 & 5585 & 5585 & 19 & $<.0001$ \\
\hline $\mathrm{F} \times \mathrm{S} \times \mathrm{C}$ & 1 & 2243 & 2243 & 8 & 0.006 \\
\hline General risk perception & 1 & 4304 & 4304 & 15 & 0.0002 \\
\hline Model $^{\mathrm{b}}$ & 12 & 409083 & 34090 & 115 & $<.0001$ \\
\hline Error & 387 & 114232 & 295 & & \\
\hline Corrected total ${ }^{\mathrm{c}}$ & 399 & 523315 & & & \\
\hline
\end{tabular}

${ }^{\mathrm{a}}$ Only effects significant at $p<0.05$ are presented

${ }^{\mathrm{b}}$ The model was controlled for age, gender, race, and education level (not shown in the table due to nonsignificant effects)

${ }^{\mathrm{c}}$ Each of the 100 participants evaluated four drug labels providing total responses $(\mathrm{N})$ of 400 


\section{American Journal of Pharmaceutical Education 2016; 80 (8) Article 131.}
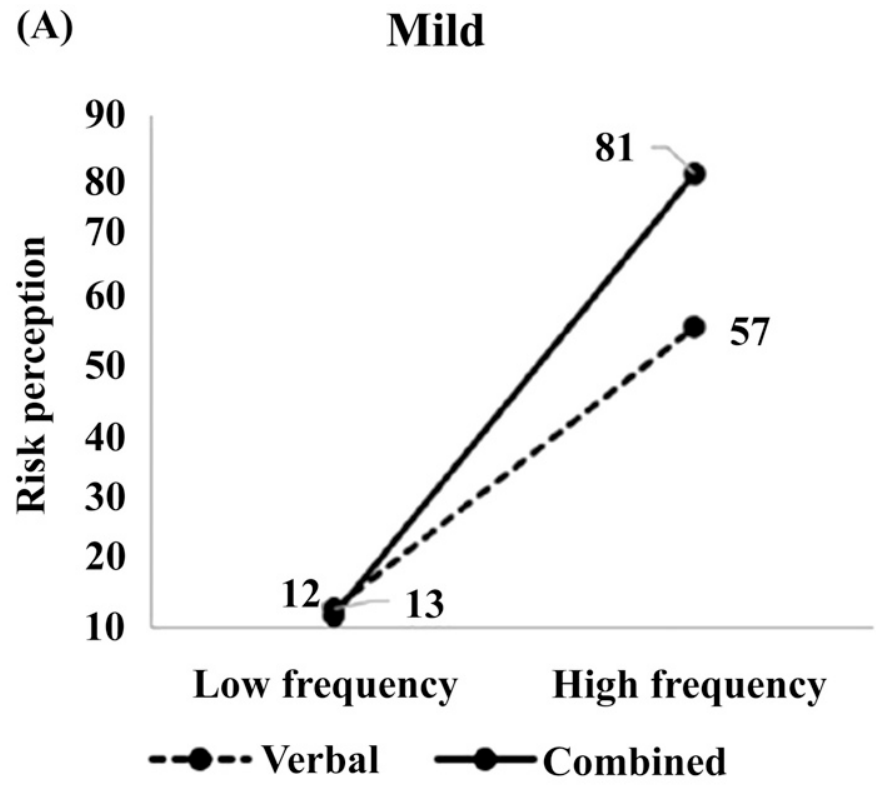

(B)

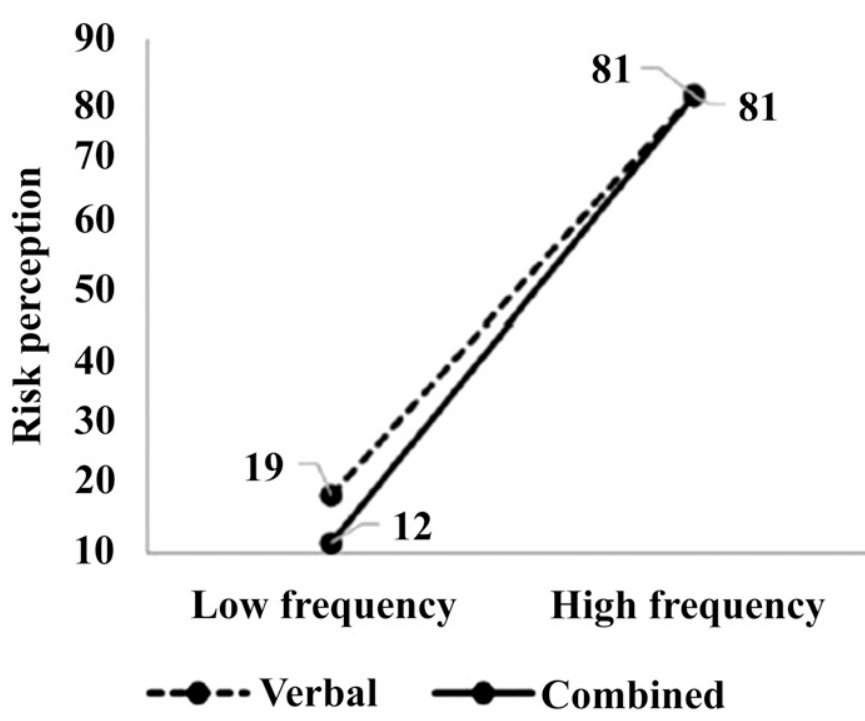

Figure 1. Interaction Effect of Communication Style and Frequency on Risk Perception Severity.

to differentiate between low and high frequency side effects. Communication style aided in understanding of the underlying context of the side effect.

The study findings shed light on to the importance of communication style and context in relaying information about medication side effects. The surveyed pharmacy students, who could be viewed as future pharmacists or consumers of medications themselves, exhibited variations in their interpretations of risk with variations in the context. Previous studies pointed out such variability, misinterpretation, and overestimation of risk with commonly used descriptors of medication side effects. ${ }^{14-20}$ These studies presented the challenges in communicating

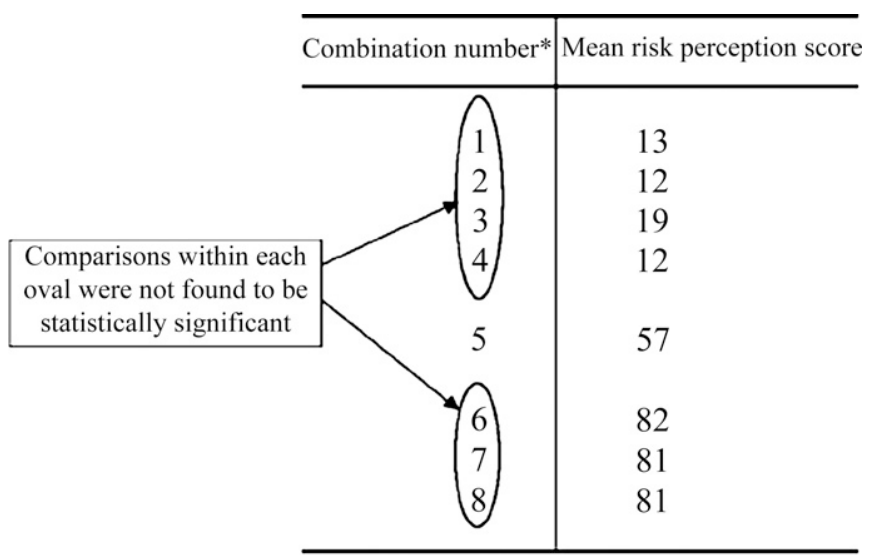

*1 =verbal $x$ low $x$ mild; $2=$ combined $x$ low $x$ mild; $3=$ verbal $x$ low $x$ severe; $\mathbf{4}=$ combined $x$ low $\times$ severe; $5=$ verbal $x$ high $\times$ mild; $6=$ combined $x$ high $\times$ mild; $7=$ verbal $x$ high $x$ severe; $8=$ combined $x$ high $x$ severe

Figure 2. Comparison of Mean Risk Perception Scores across Combinations of Communication Style (verbal/combined), Frequency (low/high) and Severity (mild/severe).

side effect risk. Thus, special attention should be paid to train pharmacy students in using language that is sufficiently flexible and considers different perspectives and changing contexts. Developing an evidence-based term list for communicating risk of medication side effects can help standardize the communication process and increase familiarity among pharmacy students and patient populations. This may, in turn, help to improve understanding and reduce noncompliance issues associated with fear of side effects.

The study should be viewed in light of its limitations. The study used a limited set of drug indications and their side effects. Reproducing the study with a broader coverage of indications and side effects may provide a better understanding of contextual influence on risk perception. The verbal descriptors used for the side effects were also limited. Future studies using a wide range of commonly used verbal descriptors may be needed. The study did not identify subjects by year in pharmacy school, so risk perception with respect to number of years spent in pharmacy education may differ. To reduce these biases, we used past education and general risk perception to control the effect. Finally, the study was conducted among pharmacy students from one pharmacy school and cannot be generalized to other pharmacy students at different institutions. Future studies may consider these factors and conduct research to evaluate the effect of years in pharmacy school, institutional curriculum, learning strategies, as well as type of verbal descriptors on risk perception communication.

\section{CONCLUSION}

Interpretations of perception of risk of experiencing side effects were influenced by communication style and contextual background among pharmacy students. 


\section{American Journal of Pharmaceutical Education 2016; 80 (8) Article 131.}

Education and training in pharmaceutical counseling should emphasize use of effective, easily understood language and terms that help in accurate estimation and communication of risk to patients.

\section{REFERENCES}

1. Focus Group Report on Consumer Knowledge About Prescription Drug Risks and Benefits. 2010.

2. Berry D, Michas I, Bersellini E. Communicating information about medication side effects: effects on satisfaction, perceived risk to health, and intention to comply. Psychology and Health. 2002;17 (3):247-267.

3. Visschers VH, Meertens RM, Passchier WW, de Vries NN. Probability information in risk communication: a review of the research literature. Risk Anal. 2009;29(2):267-287.

4. Hendrickx L, Vlek C, Oppewal H. Relative importance of scenario information and frequency information in the judgment of risk. Acta Psychologica. 1989;72(1):41-63.

5. Berry DC, Raynor DK, Knapp P, Bersellini E. Patients' understanding of risk associated with medication use. Drug Safety. 2003;26(1):1-11.

6. Hoffrage U, Gigerenzer G. Using natural frequencies to improve diagnostic inferences. Acad Med. 1998;73(5):538-540.

7. Gigerenzer G, Edwards A. Simple tools for understanding risks: from innumeracy to insight. BMJ: British Medical Journal. 2003;327 (7417):741.

8. Erev I, Cohen BL. Verbal versus numerical probabilities: Efficiency, biases, and the preference paradox. Organizational behavior and human decision processes. 1990;45(1):1-18.

9. ASHP guidelines on pharmacist-conducted patient education and counseling. Am J Health Syst Pharm. 1997;54(4):431-434.
10. Antworth A, Maffeo C. Pharmacy student self-perception of weight and relationship to counseling patients on lifestyle modification. Am J Pharm Educ. 2014;78(2):Article 35.

11. Ahmed SI, Hassali MA, Aziz NA. An assessment of the knowledge, attitudes, and risk perceptions of pharmacy students regarding HIV/AIDS. Am J Pharm Educ. 2009;73(1):Article 15. 12. Dyck A, Deschamps M, Taylor J. Pharmacists' discussions of medication side effects: a descriptive study. Patient education and counseling. 2005;56(1):21-27.

13. Weber EU, Hilton DJ. Contextual effects in the interpretations of probability words: Perceived base rate and severity of events. J. Exp. Psychol. Hum. Percept. Perform. 1990;16(4):781.

14. Dickinson D, Raynor DK, Duman M. Patient information leaflets for medicines: using consumer testing to determine the most effective design. Patient Educ Couns. 2001;43(2):147-159.

15. Bryant GD, Norman GR. Expressions of probability: words and numbers. N Engl J Med. 1980;302(7):411.

16. Mazur DJ, Merz JF. Patients' interpretations of verbal expressions of probability: implications for securing informed consent to medical interventions. Behav Sci Law. 1994;12(4):417426.

17. Reagan RT, Mosteller F, Youtz C. Quantitative meanings of verbal probability expressions. J Appl Psychol. 1989;74(3):433-442. 18. Berry D, Knapp P, Raynor T. "Is 15 per cent very common? Informing people about the risks of medication side effects." International Journal of Pharmacy Practice. 2002;3(10):145-151. 19. Knapp P, Raynor DK, Berry DC. Comparison of two methods of presenting risk information to patients about the side effects of medicines. Quality and safety in Health Care. 2004;13(3):176-180. 20. Knapp P, Raynor DK, Woolf E, Gardner PH, Carrigan N, McMillan B. Communicating the Risk of Side Effects to Patients. Drug Safety. 2009;32(10):837-849.

Appendix 1. Sample Drug Information Boxes for Drug A

Verbal Communication Style

Drug A

What is this drug for?

Drug A is used to prevent stroke after certain surgical procedures.

What are some side effects of this drug?

Drug A can rarely cause stomach bleeding.
Combined Communication Style

Drug A

What is this drug for?

Drug A is used to prevent stroke after certain surgical procedures.

What are some side effects of this drug?

Drug A can rarely cause stomach bleeding. Out of 100 people taking Drug A for one year, 2 will experience stomach bleeding. 